\title{
One-Year Outcomes of Second-Generation Trabecular Micro-Bypass Stents (iStent Inject) Implantation with Cataract Surgery in Different Glaucoma Subtypes and Severities
}

\author{
Ali Salimi (D) · Julie Lapointe $\cdot$ Paul Harasymowycz
}

Received: August 9, 2019 / Published online: September 19, 2019

(C) The Author(s) 2019

\begin{abstract}
Purpose: To assess the 1-year efficacy and safety of the implantation of two second-generation trabecular micro-bypass stents (iStent Inject $^{\circledR}$ ) with concomitant cataract surgery in various subtypes and severities of glaucoma.

Methods: This single-surgeon, consecutive case series from a Canadian academic ophthalmology center included subjects with cataract, glaucoma, and the need to reduce intraocular pressure (IOP) and/or medications. The 12-month outcomes included mean IOP and medication burden as well as the proportions of eyes with IOP $\leq 18, \leq 15$, and $\leq 12 \mathrm{mmHg}$ compared to baseline. Other measures included
\end{abstract}

Enhanced Digital Features To view enhanced digital features for this article go to https://doi.org/10.6084/ m9.figshare.9762590.

A. Salimi

Faculty of Medicine, McGill University, Montreal, QC, Canada

A. Salimi $\cdot$ J. Lapointe $\cdot$ P. Harasymowycz $(\varangle)$ Montreal Glaucoma Institute, Montreal, QC, Canada

e-mail: pavloh@igmtl.com

J. Lapointe

Department of Ophthalmology, University of

Sherbrooke, Sherbrooke, QC, Canada

P. Harasymowycz

Department of Ophthalmology, University of

Montreal, Montreal, QC, Canada corrected distance visual acuity (CDVA), cup-todisc ratio (CDR), visual field mean deviation (VF $\mathrm{MD})$, retinal nerve fiber layer (RNFL) thickness, ganglion cell inner plexiform layer (GCIPL) thickness, and adverse events.

Results: In 118 eyes, mean IOP reduced from $17.00 \pm 3.82 \mathrm{mmHg}$ preoperatively to $13.97 \pm 2.65 \mathrm{mmHg}$ at the 12-month follow-up mark $(17.8 \%$ reduction, $p<0.001)$, and mean medication burden decreased from $2.31 \pm 1.33$ preoperatively to $1.03 \pm 1.10$ medications $(56 \%$ reduction, $p<0.001$ ). After 12 months, $93 \%$ of eyes achieved IOP $\leq 18 \mathrm{mmHg}$ (versus 69\% preoperatively), $70 \%$ of eyes achieved IOP $\leq 15 \mathrm{mmHg}$ (versus $42 \%$ preoperatively), and $29 \%$ of eyes achieved IOP $\leq 12 \mathrm{mmHg}$ (versus $7 \%$ preoperatively). For all eyes, topical medications were either maintained or decreased from baseline, with $\geq 1$ medication eliminated from the preoperative regimen for $83 \%$ of eyes and $\geq 2$ medications eliminated for $36 \%$ of eyes. Visual acuity improved significantly, consistent with expectations for cataract surgery, while CDR, VF MD, and RNFL and GCIPL thicknesses remained stable. Safety was favorable, with no intraoperative complications and few transient adverse events postoperatively.

Conclusion: iStent Inject implantation with cataract surgery safely reduced IOP and medication burden in a real-world clinical population with mild to severe glaucoma, and 
stabilized visual field, as well as RNFL and GCIPL thicknesses on OCT.

Funding: The Rapid Service Fees were funded by Glaukos Corporation.

Keywords: Cataract; iStent Inject; Glaucoma; Micro-invasive glaucoma surgery (MIGS); Stent; Trabecular micro-bypass

\section{INTRODUCTION}

Glaucoma is a leading cause of visual disability and irreversible blindness that affects approximately 70 million people worldwide $[1,2]$. Current glaucoma treatment options are focused on lowering intraocular pressure (IOP), with the ultimate goal of preserving optic nerve health and preventing visual field (VF) loss. Indeed, growing evidence has highlighted the association between an elevated IOP and the thinning of the retinal nerve fiber layer (RNFL) and the ganglion cell inner plexiform layer (GCIPL) [3]. Available therapeutic options to decrease IOP include medication, laser treatment, micro-invasive glaucoma surgery (MIGS) implants or trabecular excision, glaucoma drainage devices, or traditional incisional filtering surgery.

Pharmacological treatment, however, is limited by poor adherence, local and systemic side effects, costs, and difficulties with instillation [4-6]. Laser trabeculoplasty is a relatively conservative intervention for mild IOP reduction, but it does induce inflammation and has a limited interval of IOP reduction [7]. Traditional incisional filtering surgeries are usually regarded as the best way to quickly and substantially drop the IOP, but they are associated with significant complications such as hypotony, choroidal detachment, bleb infection or leak, and endophthalmitis [8-10]. Given these risks, several of which may cumulate over time, filtering procedures are often reserved for cases resistant to topical ocular hypotensive drugs and laser treatments.

If the risks of filtering surgery outweigh the potential benefits, ab interno micro-invasive glaucoma surgery (MIGS) may offer a propitious alternative. In the past decade, the glaucoma field has seen the increasing use of MIGS procedures, with the first US FDA-approved MIGS implant being the iStent trabecular micro-bypass (iStent ${ }^{\circledR}$, Glaukos Corporation, San Clemente, CA, USA) $[11,12]$. This stent allows circumvention of the damaged trabecular meshwork by creating a direct route from the anterior chamber to Schlemm's canal. Considerable evidence has shown the stents' ability to provide meaningful postoperative reductions in IOP and medication burden for up to 5 years with or without concomitant cataract surgery, and in various subtypes and severities of glaucoma [11-17]. Moreover, these benefits have occurred with favorable safety and minimal disruption to ocular structures.

More recently, the second-generation iStent Inject ${ }^{\circledR}$ trabecular micro-bypass stent (Glaukos Corporation) has been developed to reduce IOP in a manner similar to the original iStent. Like the first-generation iStent, iStent Inject stents are made of biocompatible, medical-grade titanium. The iStent Inject device consists of an injector preloaded with two heparin-coated titanium stents, each having a central lumen and four side outlets to allow for multidirectional aqueous outflow. The stents are placed $a b$ interno through two separate regions of the trabecular meshwork into Schlemm's canal, where aqueous humor subsequently flows into collector channels en route out of the eye. The two stents are able to tap into up to 6 clock hours (i.e., half of the total span of the angle) of collector channels. The placement of stents in two separate areas increases accessibility to more collector channels for aqueous humor outflow, potentially enabling a greater IOP reduction.

Previous studies have highlighted the efficacy of the iStent Inject with and without concomitant cataract surgery in reducing the IOP and medication burden [18-27]. In this study, we present a large consecutive case series of eyes undergoing iStent Inject implantation with concomitant cataract surgery by a single surgeon at an academic ophthalmology center in Montreal, Canada. With a postoperative followup of 12 months, this study provides real-world data on IOP and medication reduction while also reporting on safety outcomes such as 
CDVA, VF, and RNFL and GCIPL thicknesses in eyes with various types and severities of glaucoma.

\section{METHODS}

\section{Study Design and Eligibility Criteria}

This single-surgeon, longitudinal consecutive case series assessed 12 months' worth of outcomes following iStent Inject implantation with cataract surgery in eyes affected by various types and severities of glaucoma. Preoperative inclusion criteria allowed for any operated patients with cataract and coexisting glaucoma [including primary open-angle (POAG), primary angle closure (PACG), normal-tension (NTG), pseudoexfoliative (PXFG), and pigmentary (PG) glaucoma]; with glaucomatous damage on retinal nerve fiber layer imaging, ganglion cell analysis, or VF; with a corrected distance visual acuity (CDVA) of 20/200 or better; with trabecular meshwork visible in the nasal angle on gonioscopy; and with the need for IOP or medication reduction. Any glaucoma severity was allowed, with severity classified according to the Hodapp-Anderson-Parrish visual field criteria (mild: VF MD no worse than $-6 \mathrm{~dB}$; moderate: VF MD worse than $-6 \mathrm{~dB}$ but no worse than $-12 \mathrm{~dB}$; severe: VF MD worse than $-12 \mathrm{~dB}$ ) [28].

Eyes were ineligible for surgery if they had nasal peripheral anterior synechiae or disorders inhibiting visualization of the nasal angle; elevated episcleral venous pressure; and chronic or acute ocular inflammation. The study was approved by the Ethics Committee of the Hôpital Maisonneuve-Rosemont in Montreal, Canada. All procedures performed were in accordance with the 1964 Declaration of Helsinki and its later amendments or comparable ethical standards. Written informed consent was obtained from all individual participants included in the study. Although analysis of this dataset was originally retrospective, patients were followed prospectively toward the end of the follow-up period with the intent of compiling a prospective case series to study longitudinal outcomes. At the time of surgery and publication of this report, iStent Inject was approved for POAG patients with mild-tomoderate disease severity. For this reason, a subgroup analysis was completed specifically for the eyes that matched those criteria $(n=53)$.

\section{Stent and Surgical Technique}

The iStent Inject trabecular micro-bypass system is preloaded with two biocompatible, heparin-coated, titanium stents. Each micro-scale stent is designed for retention in the trabecular meshwork and has multiple lateral outlet lumina to allow for aqueous humor outflow from the anterior chamber, thereby decreasing IOP. The symmetrically designed structure of the stent enables it to be implanted in either the right or left eye, and by either the right or left hand of the surgeon. At the start of each surgery, in order to ensure maximal proximity of the stent to the collector channel ostia, two separate areas located at least two clock hours apart in the nasal angle with greater trabecular pigmentation [29] or with evidence of focal blood reflux [30] were identified and marked at the corneal limbus using a sterile marker. A standard phacoemulsification incision was made, through which the injector was advanced $\mathrm{ab}$ interno to the nasal trabecular meshwork. The surgeon then activated a release button to implant the stents into the trabecular meshwork adjacent to the limbal markings. This was followed by standard phacoemulsification cataract surgery through the same clear corneal incision. After surgery, subjects received oral acetazolamide (500 $\mathrm{mg}$ on the first evening), topical moxifloxacin (3 times per day for 1 week), topical nepafenac ( 3 times per day for 1 month), and a 2-week regimen of topical loteprednol (tapered from 4 times per day). Subjects' preexisting glaucoma medication regimens were discontinued or modified based on disease severity and postoperative IOP.

\section{Study Outcomes and Data Analysis}

Efficacy measures observed over 12 months postoperatively included mean IOP and medication usage, as well as proportional analyses of 
eyes with $\mathrm{IOP} \leq 18$, $\leq 15$, and $\leq 12 \mathrm{mmHg}$; eyes on 0 or $\geq 2$ medications; and eyes with reductions of $\geq 0, \geq 1, \quad$ or $\geq 2$ medication(s) versus their preoperative state. Safety measures included noting any intra- and postoperative adverse events, corrected-distance visual acuity (CDVA), cup-to-disc ratio (CDR), visual field mean deviation (VF MD), increase in glaucoma medications for uncontrolled IOP, and secondary glaucoma surgeries.

Efficacy and safety measures were evaluated over the course of 12 months using repeatedmeasure analysis of variance (ANOVA). For variables with a significant 12-month change, linear regression models were used to assess predictors of response. All statistical analyses were performed using SPSS Statistics 25.0 (IBM, Armonk, NY, USA), with significance set at $p<0.05$.

\section{RESULTS}

\section{Demographics}

A total of 118 eyes from 71 patients with a 12-month follow-up dataset are presented in this consecutive case series. Diagnoses consisted of POAG $(n=64)$, PACG $(n=23)$, NTG $(n=16)$, PXFG $(n=10)$, and PG $(n=5)$. Disease severity did not differ across different glaucoma subtypes $(p=0.169)$. The majority of the eyes $(81 \%)$ had mild or moderate glaucoma, while $19 \%$ had severe disease. Half of the eyes had no prior glaucoma surgery, $30 \%$ had prior incisional glaucoma surgery, and $20 \%$ had previous laser peripheral iridotomy, which consisted of all PACG eyes $(n=23)$ as well as a PXFG eye with narrow angles. The mean preoperative IOP was $17.00 \pm 3.82 \mathrm{mmHg}$ on a mean of $2.31 \pm 1.33$ medications. The demographics and baseline clinical characteristics of the patients are presented in Table 1.

\section{Efficacy}

At the 12-month mark following cataract surgery combined with implantation of iStent Inject, both IOP and medication burden
Table 1 Demographic and preoperative ocular characteristics

\begin{tabular}{|c|c|}
\hline Variable & $\begin{array}{l}N=118 \text { eyes of } 71 \\
\text { subjects }\end{array}$ \\
\hline Age at time of surgery (years) & $68.56 \pm 8.74$ \\
\hline Gender (male: female) & $59: 59$ \\
\hline Eye (OD: OS) & $56: 62$ \\
\hline \multicolumn{2}{|c|}{ History of prior glaucoma interventions $\%(n)$} \\
\hline Laser peripheral iridotomy & $20.3 \%(24 / 118)$ \\
\hline Selective laser trabeculoplasty & $28.0 \%(33 / 118)$ \\
\hline Trabeculectomy & $1.7 \%(2 / 118)$ \\
\hline \multicolumn{2}{|l|}{ Type of glaucoma } \\
\hline Primary open angle glaucoma & $54.2 \%(64 / 118)$ \\
\hline Primary angle closure glaucoma & $19.5 \%(23 / 118)$ \\
\hline Normal-tension glaucoma & $13.6 \%(16 / 118)$ \\
\hline Pseudoexfoliative glaucoma & $8.5 \%(10 / 118)$ \\
\hline Pigmentary glaucoma & $4.2 \%(5 / 118)$ \\
\hline Intraocular pressure $(\mathrm{mmHg})$ & $17.00 \pm 3.82$ \\
\hline Glaucoma medications & $2.31 \pm 1.33$ \\
\hline $\begin{array}{l}\text { Corrected distance visual acuity } \\
\text { (LogMAR) }\end{array}$ & $0.17 \pm 0.22$ \\
\hline Cup to disc ratio & $0.70 \pm 0.12$ \\
\hline Visual field-mean deviation $(\mathrm{dB})$ & $-6.57 \pm 6.09$ \\
\hline $\begin{array}{l}\text { Visual field—pattern standard } \\
\text { deviation }(\mathrm{dB})\end{array}$ & $4.59 \pm 2.78$ \\
\hline $\begin{array}{l}\text { Retinal nerve fiber layer thickness } \\
(\mu \mathrm{m})\end{array}$ & $71.10 \pm 12.62$ \\
\hline $\begin{array}{l}\text { Ganglion cell inner plexiform layer } \\
\text { thickness }(\mu \mathrm{m})\end{array}$ & $67.72 \pm 9.12$ \\
\hline
\end{tabular}

showed significant reductions that had been maintained throughout follow-up. Mean IOP reduced by $17.8 \%$, from $17.00 \pm 3.83 \mathrm{mmHg}$ to $13.97 \pm 2.65$ with a large effect size $(p<0.001$, $\eta^{2}=0.411,95 \%$ confidence interval (CI) -3.70 to -2.37 ) (Fig. 1, Table 2). As illustrated in 


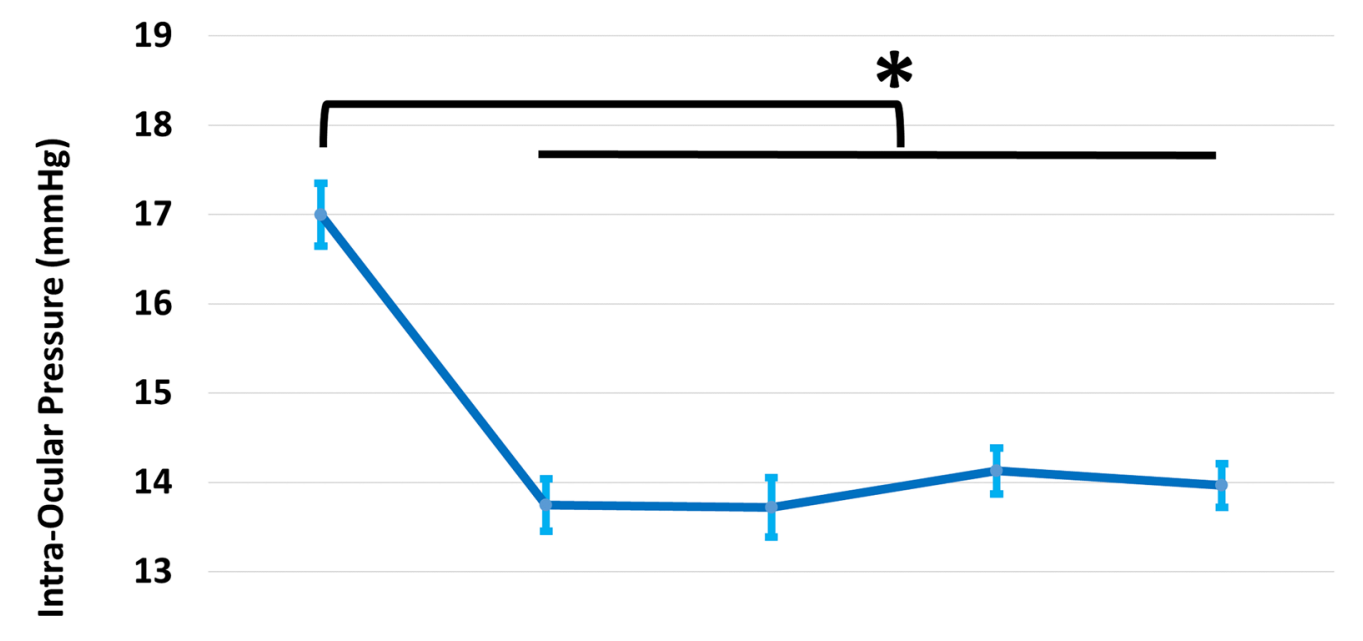

12

\begin{tabular}{|c|c|c|c|c|c|}
\multicolumn{1}{c|}{11} & Pre-Op & $1 \mathrm{M} \mathrm{F} / \mathrm{U}$ & $3 \mathrm{M} \mathrm{F} / \mathrm{U}$ & $6 \mathrm{M} \mathrm{F} / \mathrm{U}$ & $12 \mathrm{M} \mathrm{F/U}$ \\
\cline { 2 - 6 } \multicolumn{1}{c|}{$\mathrm{IOP}(\mathrm{mmHg})$} & 17.00 & 13.75 & 13.72 & 14.13 & 13.97 \\
\hline
\end{tabular}

Fig. 1 Change in average intraocular pressure $(n=118)$. Following cataract surgery combined with implantation of iStent Inject, mean intraocular pressure significantly decreased and was maintained throughout follow-up $(p<0.001)$. Asterisk signifies statistical differences at $p<0.05$; bars demonstrate standard error of the mean; $I O P$ intraocular pressure
Fig. 2, approximately 93\% of eyes achieved IOP $\leq 18 \mathrm{mmHg}$ at the 12 -month mark (versus $69 \%$ preoperatively), $70 \%$ of eyes achieved IOP $\leq 15 \mathrm{mmHg}$ (versus $42 \%$ preoperatively), and $29 \%$ of eyes achieved IOP $\leq 12 \mathrm{mmHg}$, versus $7 \%$ preoperatively.

Mean medication burden decreased by $56 \%$ to $1.03 \pm 1.10$ medications from the preoperative mean of $2.31 \pm 1.33\left(p<0.001, \eta^{2}=0.680\right.$, $95 \%$ CI -1.45 to -1.13 ) (Fig. 3 , Table 2). All 118 eyes either maintained or decreased their topical medication burden compared to their preoperative usage, with $\geq 1$ medication eliminated from the original regimen for $83 \%$ of the eyes and $\geq 2$ medications eliminated for $36 \%$ of the eyes (Fig. 4). At 12 months postoperatively, $47 \%$ of the eyes were medication-free compared to $4 \%$ preoperatively, and only $40 \%$ of the eyes were on $\geq 2$ medications, as opposed to $67 \%$ preoperatively.

A linear regression model was created to identify predictors of postoperative IOP improvement (Table 3). The model showed that greater baseline preoperative IOP $(\beta=-0.772$, $p<0.001)$ and thinner corneas $(\beta=0.148$, $p=0.042$ ) were associated with a larger postoperative IOP reduction. A second model was created to identify predictors of postoperative medication reduction $\left(R^{2}=0.361\right.$, adjusted $\left.R^{2}=0.376, p<0.001\right)$, which showed that a higher number of glaucoma medications at baseline was associated with a greater reduction in medication burden postoperatively $(\beta=-0.629, p<0.001)$. Differences in age, sex, type, and severity of glaucoma and in prior history of glaucoma surgery did not account for the amount of IOP or medication reduction in either model.

As iStent is indicated for POAG with mild to moderate severity, we performed a subgroup analysis for eyes that met that criterion. Fiftythree eyes with an average age of $70.26 \pm 8.74$ years and a mean baseline IOP of $17.28 \pm 3.55 \mathrm{mmHg}$ on an average of $2.09 \pm 1.15$ antiglaucoma medications were included. The subgroup outcomes were similar 
Table 2 Twelve-month outcomes in efficacy and safety measures $(N=118$ eyes of 71 subjects $)$

\begin{tabular}{lcccc}
\hline Variable & Baseline & 12-Month follow-up & $\boldsymbol{\eta}^{\mathbf{2}}$ & $\boldsymbol{p}$ value \\
\hline Intraocular pressure $(\mathrm{mmHg})$ & $17.00 \pm 3.82$ & $13.97 \pm 2.65$ & 0.411 & $<0.001^{*}$ \\
Glaucoma medications & $2.31 \pm 1.33$ & $1.03 \pm 1.10$ & 0.680 & $<0.001^{*}$ \\
Corrected distance visual acuity (LogMAR) & $0.17 \pm 0.22$ & $0.10 \pm 0.17$ & 0.163 & $<0.001^{*}$ \\
Cup to disc ratio & $0.70 \pm 0.12$ & $0.69 \pm 0.13$ & 0.023 & 0.113 \\
Visual field-mean deviation $(\mathrm{dB})$ & $-6.57 \pm 6.09$ & $-6.49 \pm 6.04$ & 0.001 & 0.729 \\
Visual field-pattern standard deviation $(\mathrm{dB})$ & $4.59 \pm 2.78$ & $4.67 \pm 2.84$ & 0.003 & 0.567 \\
Retinal nerve fiber layer thickness $(\mu \mathrm{m})$ & $71.10 \pm 12.62$ & $71.61 \pm 12.94$ & 0.016 & 0.184 \\
Ganglion cell inner plexiform layer thickness $(\mu \mathrm{m})$ & $67.72 \pm 9.12$ & $67.79 \pm 9.88$ & 0.000 & 0.906 \\
\hline
\end{tabular}

Mean \pm standard deviations are presented and statistically compared

*Denotes statistical significance

to those of the overall cohort: IOP decreased by $19.0 \%$ to $14.00 \pm 2.47 \mathrm{mmHg} \quad(p<0.001$, $\eta^{2}=0.482,95 \% \mathrm{CI}-4.23$ to -2.34$)$ and medication burden decreased by $62.2 \%$ to $0.79 \pm 0.93 \quad\left(p<0.001, \quad \eta^{2}=0.730, \quad 95 \% \quad\right.$ CI -1.52 to -1.08$)$.

\section{Safety Measurements}

All eyes were successfully implanted with two iStent Inject stents with no intraoperative complications. Postoperatively, CDVA improved significantly, consistent with the expectations for cataract surgery $(p<0.001$, $\eta^{2}=0.163,95 \%$ CI $\left.0.045-0.110\right)$. Additionally, stability was maintained for 12 months in patients' CDR $(p=0.113)$, VF MD $(p=0.729)$, and thicknesses of the retinal nerve fiber layer $(p=0.184)$ and ganglion cell inner plexiform layer $(p=0.906)$.

Relatively few adverse events occurred and all were transient, had no sight-threatening sequelae, and were managed conservatively. Adverse events included IOP spike (defined as IOP greater than 50\% above baseline [31]) in $11 \%$ of the eyes within the first two postoperative weeks, which can be attributed to the postoperative steroid regimen, and microhyphema in 5\% of the eyes within the first $24 \mathrm{~h}$ after surgery, which can be expected with ocular

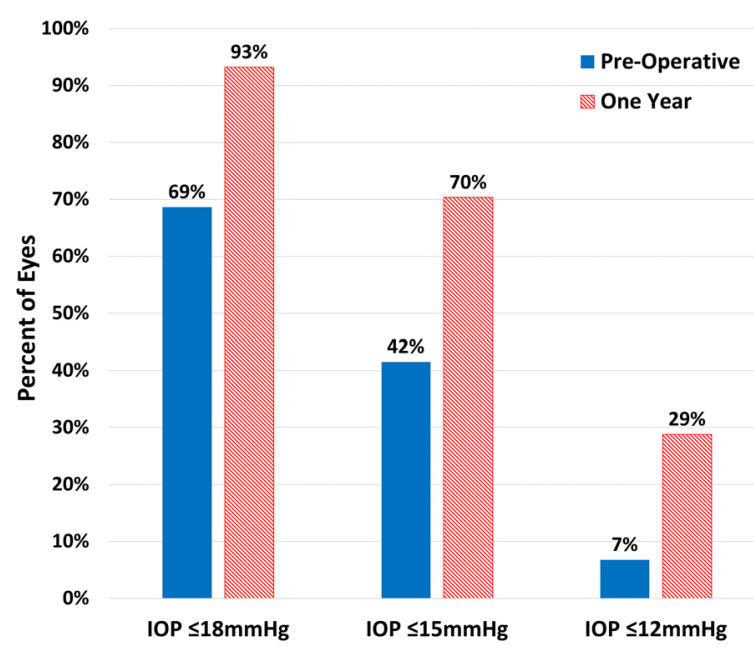

Fig. 2 Percentages of eyes with IOP $\leq 18, \leq 15$, and $\leq 12 \mathrm{mmHg}(n=118)$ preoperatively and at 1 -year follow-up. Solid bars and diagonally hashed bars represent preoperative and 1-year follow-up data, respectively. IOP intraocular pressure

surgery. Other events that are common after cataract surgery (and are unrelated to stents) included transient corneal edema $24 \mathrm{~h}$ after surgery in $10 \%$ of the eyes, posterior capsular opacification within the first six postoperative months in $8 \%$ of the eyes, and rebound iritis in $6 \%$ of the eyes within the first postoperative month. Notably, there were no reports of hypotony, choroidal detachment, stent 


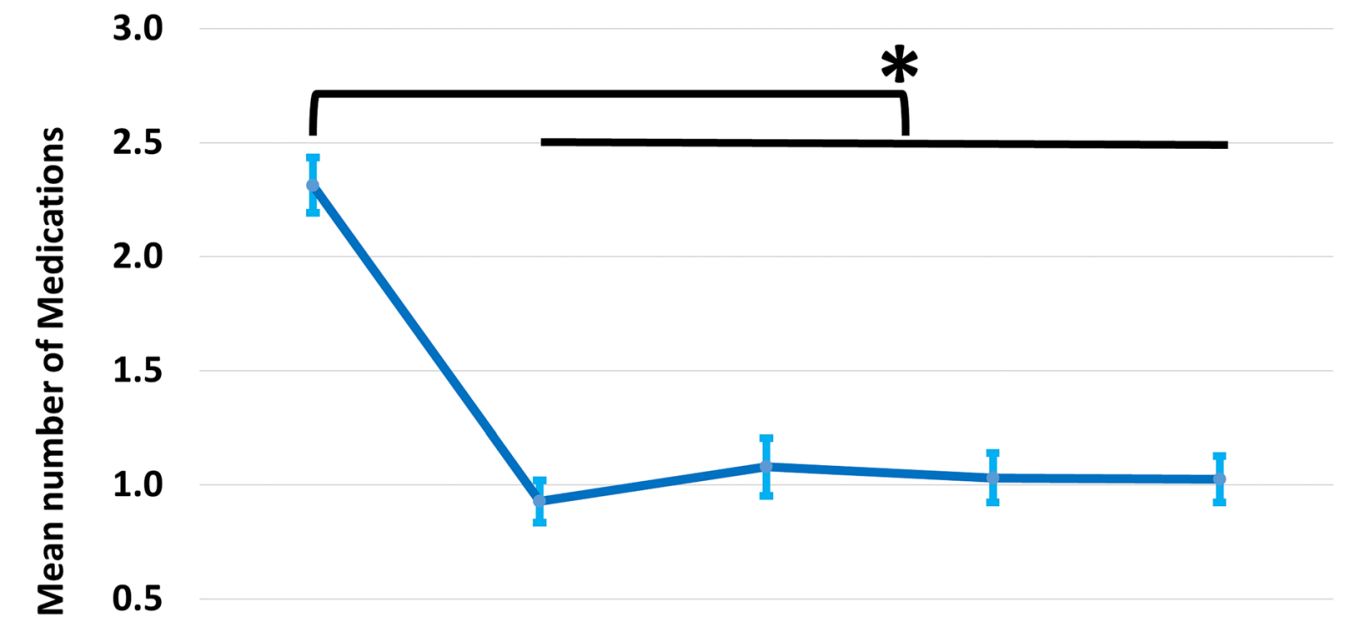

\begin{tabular}{|c|c|c|c|c|c|}
\hline & Pre-Op & $1 M F / U$ & $3 M F / U$ & $6 M F / U$ & $12 \mathrm{M} \mathrm{F} / \mathrm{U}$ \\
\hline Medication & 2.31 & 0.93 & 1.08 & 1.03 & 1.03 \\
\hline
\end{tabular}

Fig. 3 Change in mean number of glaucoma medications $(n=118)$. Following cataract surgery combined with implantation of iStent Inject, the mean number of medications significantly decreased, and this was

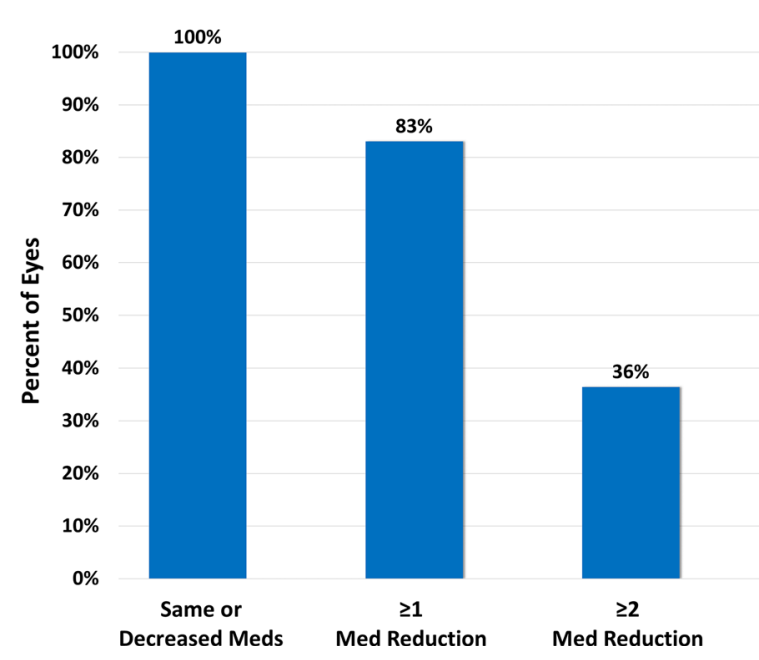

Fig. 4 Proportions of the eyes with reductions of $\geq 0$, $\geq 1$, or $\geq 2$ medication(s) at $\mathrm{t} 12$ months. Solid bars represent the 1-year follow-up data. Med medication

obstruction, stent extrusion, peripheral anterior synechiae (PAS), myopic shift, or endophthalmitis. During follow-up, only three eyes underwent selective laser trabeculoplasty: one for persistent elevated IOP and two for maintained throughout follow-up $(p<0.001)$. Asterisk signifies statistical differences at $p<0.05$; bars demonstrate standard error of the mean

intolerance to antiglaucoma drops and the need for IOP reduction without pharmacotherapy. Importantly, no increase in the number of antiglaucoma medications was documented postoperatively, and no eye needed incisional secondary glaucoma surgeries during follow-up.

\section{DISCUSSION}

A surgical device to treat glaucoma-especially a device with favorable safety and durable effectiveness-has many advantages over medication therapy. Suboptimal medication adherence can be a significant obstacle to achieving disease control, and poor adherence has been shown to promote disease progression [32-35]. Additionally, even in the setting of perfect adherence, normal diurnal variations in intraocular drug activity may permit IOP fluctuations that increase the risk of optic nerve damage [36, 37]. Comparatively, surgical treatments have been proven to have less IOP variability than medications $[38,39]$. Thus, an implanted device that delivers a consistent IOP reduction 
Table 3 Linear regression models for baseline predictors of improvements in IOP and medication burden at 1 year postoperatively ( $N=118$ eyes of 71 subjects)

\begin{tabular}{|c|c|c|c|c|c|c|}
\hline Independent variable & $\boldsymbol{B}$ & $\beta$ & $t$ value & $p$ value & $95 \% \mathrm{CI}$ & \\
\hline \multicolumn{7}{|c|}{ Intraocular pressure $\left(R^{2}=0.591\right.$, adjusted $\left.R^{2}=0.557, p<0.001\right)$} \\
\hline Age (years) & -0.004 & -0.011 & -0.143 & 0.887 & -0.067 & 0.058 \\
\hline Sex & 0.130 & 0.018 & 0.258 & 0.797 & -0.866 & 1.125 \\
\hline Glaucoma subtype & 0.112 & 0.037 & 0.538 & 0.592 & -0.301 & 0.525 \\
\hline Previous glaucoma intervention & -0.139 & -0.019 & -0.263 & 0.793 & -1.188 & 0.910 \\
\hline Central corneal thickness $(\mu \mathrm{m})$ & 0.014 & 0.148 & 2.063 & $0.042^{*}$ & 0.001 & 0.027 \\
\hline Baseline intraocular pressure $(\mathrm{mmHg})$ & -0.737 & -0.772 & -11.459 & $<0.001^{*}$ & -0.864 & -0.609 \\
\hline Baseline medication burden & 0.200 & 0.073 & 1.015 & 0.313 & -0.191 & 0.591 \\
\hline Visual field-mean deviation $(\mathrm{dB})$ & 0.030 & 0.052 & 0.729 & 0.468 & -0.052 & 0.112 \\
\hline \multicolumn{7}{|c|}{ Medication burden $\left(R^{2}=0.361\right.$, adjusted $\left.R^{2}=0.376, p<0.001\right)$} \\
\hline Age (years) & -0.004 & -0.038 & -0.400 & 0.690 & -0.023 & 0.015 \\
\hline Sex & 0.109 & 0.062 & 0.713 & 0.477 & -0.194 & 0.412 \\
\hline Glaucoma subtype & 0.055 & 0.075 & 0.871 & 0.386 & -0.071 & 0.181 \\
\hline Previous glaucoma intervention & 0.114 & 0.064 & 0.708 & 0.481 & -0.206 & 0.433 \\
\hline Central corneal thickness $(\mu \mathrm{m})$ & 0.000 & -0.010 & -0.111 & 0.912 & -0.004 & 0.004 \\
\hline Baseline intraocular pressure $(\mathrm{mmHg})$ & 0.028 & 0.123 & 1.455 & 0.149 & -0.010 & 0.067 \\
\hline Baseline medication burden & -0.419 & -0.629 & -6.988 & $<0.001^{*}$ & -0.539 & -0.300 \\
\hline Visual field-mean deviation $(\mathrm{dB})$ & 0.000 & -0.001 & -0.006 & 0.995 & -0.025 & 0.025 \\
\hline
\end{tabular}

$B$ regression coefficient, $\beta$ standardized coefficient beta, $C I$ confidence interval

${ }^{*}$ Denotes statistical significance

independently of external factors avoids both the adherence issues and IOP fluctuations inherent in medication usage. The safe and sustained IOP- and medication-reducing outcomes of the present study indicate that the iStent Inject trabecular micro-bypass stent is a favorable option, with cataract surgery offering an opportune moment for their implantation. Furthermore, this micro-invasive, ab interno implantation procedure preserves conjunctival tissue in case future surgery is needed.

The IOP and medication reductions of the present study are consistent with prior evaluations of second-generation iStent Inject trabecular bypass stents combined with cataract surgery for open-angle glaucoma [27, 40-42]. Mean IOP was reduced by $2.75 \mathrm{mmHg}(16.2 \%)$, a modest IOP reduction that should be considered in the context of the cohort's low mean preoperative IOP of $17.00 \mathrm{mmHg}$. Since it is lower than in most existing studies of trabecular bypass stents, the cohort's low mean preoperative IOP makes it more difficult to achieve large postoperative IOP reductions. Given that glaucoma may continue to progress in many patients despite them having an IOP in the normal range [43], and the fact that each $1 \mathrm{mmHg}$ reduction in IOP leads to a $10 \%$ reduction in the risk of glaucoma progression [44], the goal for surgery in these patients was to 
achieve an even lower IOP while also reducing the medication burden.

Most of the evidence in the literature on iStent Inject revolves around the outcomes related to POAG patients with mild to moderate severity-the population for whom this device is approved. We performed a subgroup analysis for eyes that met these criteria, but we also report outcomes of the overall dataset, including off-label use in PACG and severe glaucoma cases. The performance of the device for these indications has not been formally established in prospective randomized controlled trials, and thus additional caution was taken before undertaking device implantation in these patients. In PACG cases specifically, all eyes had a previous LPI, and adequate visualization of the nasal angle structures was ensured prior to implantation of the stent. Interestingly, the regression analyses did not show any impact of glaucoma subtype or severity on the degree of IOP and medication reduction. This lack of association suggests that iStent Inject may be effective in a wide range of glaucoma subtypes and severities, including in non-POAG cases and severe glaucoma cases. In line with our findings, other recent studies have also shown significant IOP and medication reductions in patients with narrow-angle glaucoma, PXFG, and severe glaucoma following combined iStent Inject implantation and cataract surgery $[45,46]$.

With regard to medications, the cohort exhibited a notable $67 \%$ decrease in mean number of medications-a number which is further supported by the literature, with previous studies reporting 1-year medication reductions ranging from 56 to $77 \%$ [20, 21, 40, 42]. As evidenced by our regression model, those with higher preoperative medication burdens experienced larger medication reductions postoperatively. At the level of individual patients, medications were maintained or decreased for all eyes versus their preoperative state; one medication was eliminated for $83 \%$ of the eyes, two were eliminated for $43 \%$ of the eyes, and medications were eliminated entirely for close to half of the eyes (46\%). Since medication burden is known to impact patient compliance and quality of life [47-49], this combined surgery may produce benefits beyond simply gaining better IOP control with fewer drops.

Importantly, the IOP- and medication-reducing performance was sustained for 12 months within our heterogeneous glaucoma population, which consisted of different glaucoma subtypes and severities. As such, we believe that our findings are pertinent to practicing ophthalmologists evaluating treatment options for glaucoma patients in realworld clinical settings. Finally, another unique aspect of this study is that the surgical technique included the identification of collector channels with the goal of facilitating stent placement adjacent to the episcleral vessels and optimizing outflow. This strategy is based upon prior work showing a correlation between greater episcleral venous outflow and better IOP outcomes [50]. The findings of our study are similar to those of multiple other studies in which collector channels were not specifically identified prior to stent placement [27, 40-42]. This can be interpreted in light of a variety of explanations. Our ability to assess the integrity and function of the collector channel preoperatively is limited, and it is possible that the collector channels identified were subject to distal atrophy [51]. Additionally, despite our effort to precisely identify the collector channels, we cannot rule out accuracy errors. Alternatively, considering our findings, it can be argued that iStent Inject has a beneficial impact independently of collector channel identification.

The safety profile of the iStent Inject device was highly favorable, with no intraoperative complications, relatively minimal and mild postoperative adverse events, and thus no significant sequelae. Visual acuity improved significantly, consistent with expectations for cataract surgery, and indicating that stent implantation has no untoward effects on the visual benefits of cataract surgery. Safety outcomes also showed that CDR, VF MD, and RNFL and GCIPL thicknesses remained stable postoperatively for 12 months. Given the established association between elevated IOP and thinning of the RNFL and GCIPL [3], the IOP-lowering effect of the iStent Inject may conceivably reduce the risk of neuronal 
loss. Furthermore, patients did not exhibit the side effects or adherence issues of topical medication [4-6], did not show the waning IOP- and medication-reducing effectiveness of laser trabeculoplasty [7], and did not demonstrate the safety risks seen with filtering surgery [8-10]. There were no reports of complications seen with traditional filtering surgeries or some other MIGS modalities, such as PAS, hypotony, choroidal detachment, stent obstruction, stent extrusion, stent-endothelial contact, or endophthalmitis [8-10, 52, 53]. While longer-term data are needed to confirm stability over time, these initial safety outcomes are reassuring.

This study has some limitations. The lack of a control arm limits our findings to the efficacy of the combined procedure as opposed to the efficacy of iStent Inject as a standalone procedure. To address this limitation, we focused our discussion and interpretation around comparing the results to evidence previously reported for combined procedures. Additionally, our study was limited to only one surgeon and clinical site. Although this factor limits the sample size, it favors the results by limiting bias due to intersurgeon differences in examination and surgical techniques. Consistent with most real-world clinical reports, patients did not complete a preoperative medication washout, as this is not indicated or appropriate in routine clinical practice. Outcomes included 12 months of follow-up, so future research could be done over longer periods of data collection, as well as with patients from multiple sites and/or with larger sample sizes. Future research could also include separate studies of individual glaucoma subtypes or analyses with greater numbers of participants to allow for different stratified analyses.

\section{CONCLUSION}

The present study provides clinically relevant, real-world data on the utility of second-generation iStent Inject trabecular bypass stents with cataract surgery in a heterogeneous, robustly sized patient population. The surgery led to meaningful patient-level reductions in medication burden as well as significantly lower IOP. IOP and medication reductions were sustained postoperatively for 12 months, conjunctival tissue was not disturbed, and safety was favorable. These findings, which were observed within a heterogeneous glaucoma population and thus are generalizable to many practicing ophthalmologists, suggest that iStent Inject trabecular micro-bypass implantation with phacoemulsification is a safe, consistently efficacious, tissue-preserving glaucoma treatment option for patients with cataract and various types of mild to severe glaucoma.

\section{ACKNOWLEDGEMENTS}

We thank the participants of the study.

Funding. No financial sponsorship was received for the work in this study. The Rapid Service Fees were funded by Glaukos Corporation. All authors had full access to all of the data in this study and take complete responsibility for the integrity of the data and accuracy of the data analysis.

Authorship. All named authors meet the International Committee of Medical Journal Editors (ICMJE) criteria for authorship for this article, take responsibility for the integrity of the work as a whole, and have given their approval for this version to be published.

Disclosures. Paul Harasymowycz is consultant for Allergan, Glaukos, Ivantis, J and J Vision, Santen. Ali Salimi and Julie Lapointe have nothing to disclose.

Compliance with Ethics Guidelines. The study was approved by the Ethics Committee of the Hôpital Maisonneuve-Rosemont in Montreal, Canada. All procedures performed were in accordance with the ethical standards of the institutional research committee and with the 1964 Helsinki Declaration and its later amendments or comparable ethical standards. Written informed consent was obtained from all individual participants included in the study. 
Open Access. This article is distributed under the terms of the Creative Commons Attribution-NonCommercial 4.0 International License (http://creativecommons.org/licenses/ by-nc/4.0/), which permits any noncommercial use, distribution, and reproduction in any medium, provided you give appropriate credit to the original author(s) and the source, provide a link to the Creative Commons license, and indicate if changes were made.

\section{REFERENCES}

1. Quigley HA, Broman AT. The number of people with glaucoma worldwide in 2010 and 2020. Br J Ophthalmol. 2006;90(3):262-7.

2. Tham YC, Li X, Wong TY, Quigley HA, Aung T, Cheng CY. Global prevalence of glaucoma and projections of glaucoma burden through 2040: a systematic review and meta-analysis. Ophthalmology. 2014;121(11):2081-90.

3. Choi JA, Shin HY, Park HL, Park CK. The pattern of retinal nerve fiber layer and macular ganglion cellinner plexiform layer thickness changes in glaucoma. J Ophthalmol. 2017;2017:6078365.

4. Nordstrom BL, Friedman DS, Mozaffari E, Quigley HA, Walker AM. Persistence and adherence with topical glaucoma therapy. Am J Ophthalmol. 2005;140(4):598-606.

5. Tsai JC. A comprehensive perspective on patient adherence to topical glaucoma therapy. Ophthalmology. 2009;116(11 Suppl):S30-6.

6. Newman-Casey PA, Robin AL, Blachley T, Farris K, Heisler M, Resnicow $\mathrm{K}$, et al. The most common barriers to glaucoma medication adherence: a crosssectional survey. Ophthalmology. 2015;122(7):1308-16.

7. Glaucoma Laser Trial Research Group. The Glaucoma Laser Trial (GLT) and glaucoma laser trial follow-up study: 7. Results. Am J Ophthalmol. 1995;120(6):718-31.

8. Jampel HD, Musch DC, Gillespie BW, Lichter PR, Wright MM, Guire KE, et al. Perioperative complications of trabeculectomy in the Collaborative Initial Glaucoma Treatment Study (CIGTS). Am J Ophthalmol. 2005;140(1):16-22.

9. Gedde SJ, Herndon LW, Brandt JD, Budenz DL, Feuer WJ, Schiffman JC, et al. Postoperative complications in the Tube Versus Trabeculectomy (TVT) study during 5 years of follow-up. Am J Ophthalmol. 2012;153(5):804-14.

10. Rulli E, Biagioli E, Riva I, Gambirasio G, De Simone I, Floriani I, et al. Efficacy and safety of trabeculectomy vs nonpenetrating surgical procedures: a systematic review and meta-analysis. JAMA Ophthalmol. 2013;131(12):1573-82.

11. Samuelson TW, Katz LJ, Wells JM, Duh YJ, Giamporcaro JE, Group USiS. Randomized evaluation of the trabecular micro-bypass stent with phacoemulsification in patients with glaucoma and cataract. Ophthalmology. 2011;118(3):459-67.

12. Craven ER, Katz LJ, Wells JM, Giamporcaro JE. Cataract surgery with trabecular micro-bypass stent implantation in patients with mild-to-moderate open-angle glaucoma and cataract: 2-year followup. J Cataract Refract Surg. 2012;38(8):1339-45.

13. Arriola-Villalobos P, Martinez-de-la-Casa JM, DiazValle D, Fernandez-Perez C, Garcia-Sanchez J, Garcia-Feijoo J. Combined iStent trabecular micro-bypass stent implantation and phacoemulsification for coexistent open-angle glaucoma and cataract: a long-term study. $\mathrm{Br} \quad \mathrm{J}$ Ophthalmol. 2012;96(5):645-9.

14. Fea AM, Consolandi G, Zola M, Pignata G, Cannizzo P, Lavia C, et al. Micro-bypass implantation for primary open-angle glaucoma combined with phacoemulsification: 4-year follow-up. J Ophthalmol. 2015;2015:795357.

15. Shiba D, Hosoda S, Yaguchi S, Ozeki N, Yuki K, Tsubota K. Safety and efficacy of two trabecular micro-bypass stents as the sole procedure in Japanese patients with medically uncontrolled primary open-angle glaucoma: a pilot case series. J Ophthalmol. 2017;2017:9605461.

16. Myers JS, Masood I, Hornbeak DM, Belda JI, Auffarth G, Junemann A, et al. Prospective evaluation of two iStent ${ }^{\circledR}$ trabecular stents, one iStent Supra ${ }^{\circledR}$ suprachoroidal stent, and postoperative prostaglandin in refractory glaucoma: 4-year outcomes. Adv Ther. 2018;35(3):395-407.

17. Katz LJ, Erb C, Carceller Guillamet A, Fea AM, Voskanyan L, Giamporcaro JE, et al. Long-term titrated IOP control with one, two, or three trabecular micro-bypass stents in open-angle glaucoma subjects on topical hypotensive medication: 42-month outcomes. Clin Ophthalmol. 2018;12:255-62.

18. Fea AM, Belda JI, Rekas M, Junemann A, Chang L, Pablo L, et al. Prospective unmasked randomized evaluation of the iStent inject ${ }^{\circledR}$ versus two ocular 
hypotensive agents in patients with primary openangle glaucoma. Clin Ophthalmol. 2014;8:875-82.

19. Voskanyan L, Garcia-Feijoo J, Belda JI, Fea A, Junemann A, Baudouin C, et al. Prospective, unmasked evaluation of the iStent ${ }^{\circledR}$ inject system for open-angle glaucoma: synergy trial. Adv Ther. 2014;31(2):189-201.

20. Klamann MK, Gonnermann J, Pahlitzsch M, Maier $\mathrm{AK}$, Joussen AM, Torun $\mathrm{N}$, et al. iStent inject in phakic open angle glaucoma. Graefes Arch Clin Exp Ophthalmol. 2015;253(6):941-7.

21. Arriola-Villalobos P, Martinez-de-la-Casa JM, DiazValle D, Morales-Fernandez L, Fernandez-Perez C, Garcia-Feijoo J. Glaukos iStent inject ${ }^{\circledR}$ trabecular micro-bypass implantation associated with cataract surgery in patients with coexisting cataract and open-angle glaucoma or ocular hypertension: a long-term study. J Ophthalmol. 2016;2016:1056573.

22. Lindstrom R, Lewis R, Hornbeak DM, Voskanyan L, Giamporcaro JE, Hovanesian J, et al. Outcomes following implantation of two second-generation trabecular micro-bypass stents in patients with open-angle glaucoma on one medication: 18-month follow-up. Adv Ther. 2016;33(11):2082-90.

23. Gonnermann J, Bertelmann E, Pahlitzsch M, MaierWenzel AB, Torun N, Klamann MK. Contralateral eye comparison study in MICS \& MIGS: Trabectome $^{\circledR}$ vs. iStent inject ${ }^{\circledR}$. Graefes Arch Clin Exp Ophthalmol. 2017;255(2):359-65.

24. Berdahl J, Voskanyan L, Myers JS, Hornbeak DM, Giamporcaro JE, Katz LJ, et al. Implantation of two second-generation trabecular micro-bypass stents and topical travoprost in open-angle glaucoma not controlled on two preoperative medications: 18-month follow-up. Clin Exp Ophthalmol. 2017;45(8):797-802.

25. Macher T, Haberle H, Wachter J, Thannhauser C, Aurich H, Pham DT. Trabecular microbypass stents as minimally invasive approach after conventional glaucoma filtration surgery. J Cataract Refract Surg. 2018;44(1):50-5.

26. Hengerer FH, Auffarth GU, Riffel C, Conrad-Hengerer I. Prospective, non-randomized, 36-month study of second-generation trabecular micro-bypass stents with phacoemulsification in eyes with various types of glaucoma. Ophthalmol Ther. 2018;7(2):405-15.

27. Guedes RAP, Gravina DM, Lake JC, Guedes VMP, Chaoubah A. Intermediate results of iStent or iStent inject implantation combined with cataract surgery in a real-world setting: a longitudinal retrospective study. Ophthalmol Ther. 2019;8(1):87-100.

28. Hodapp E, Parrish RK, Anderson DR. Clinical decisions in glaucoma. St. Louis: Mosby Inc; 1993.

29. Hann CR, Fautsch MP. Preferential fluid flow in the human trabecular meshwork near collector channels. Investig Ophthalmol Vis Sci. 2009;50(4):1692-7.

30. Saheb H, Ahmed II. Micro-invasive glaucoma surgery: current perspectives and future directions. Curr Opin Ophthalmol. 2012;23(2):96-104.

31. Salimi A, Winter A, Li C, Harasymowycz P, Saheb H. Effect of topical corticosteroids on early postoperative intraocular pressure following combined cataract and trabecular microbypass surgery. J Ocul Pharmacol Ther. 2019;35(7):413-20. https://doi. org/10.1089/jop.2019.0019.

32. Sleath B, Blalock S, Covert D, Stone JL, Skinner AC, Muir K, et al. The relationship between glaucoma medication adherence, eye drop technique, and visual field defect severity. Ophthalmology. 2011;118(12):2398-402.

33. Stewart WC, Chorak RP, Hunt HH, Sethuraman G. Factors associated with visual loss in patients with advanced glaucomatous changes in the optic nerve head. Am J Ophthalmol. 1993;116(2):176-81.

34. Rossi GC, Pasinetti GM, Scudeller L, Radaelli R, Bianchi PE. Do adherence rates and glaucomatous visual field progression correlate? Eur J Ophthalmol. 2011;21(4):410-4.

35. Paula JS, Furtado JM, Santos AS, Coelho Rde M, Rocha EM, Rodrigues Mde L. Risk factors for blindness in patients with open-angle glaucoma followed-up for at least 15 years. Arq Bras Oftalmol. 2012;75(4):243-6.

36. Nouri-Mahdavi K, Medeiros FA, Weinreb RN. Fluctuation of intraocular pressure as a predictor of visual field progression. Arch Ophthalmol. 2008;126(8):1168-9 (author reply 1169-70).

37. Fechtner RD, Voskanyan L, Vold SD, Tetz M, Auffarth G, Masood I, et al. Five-year, prospective, randomized, multi-surgeon trial of two trabecular bypass stents versus prostaglandin for newly diagnosed open-angle glaucoma. Ophthalmol Glaucoma. 2019;2(3):156-66.

38. Posarelli C, Ortenzio P, Ferreras A, Toro MD, Passani A, Loiudice $P$, et al. Twenty-four-hour contact lens sensor monitoring of aqueous humor dynamics in surgically or medically treated glaucoma patients. J Ophthalmol. 2019;2019:9890831. 
39. Muniesa MJ, Ezpeleta J, Benitez I. Fluctuations of the intraocular pressure in medically versus surgically treated glaucoma patients by a contact lens sensor. Am J Ophthalmol. 2019;203:1-11.

40. Hooshmand J, Rothschild P, Allen P, Kerr NM, Vote BJ, Toh T. Minimally invasive glaucoma surgery: comparison of iStent with iStent inject in primary open angle glaucoma. Clin Exp Ophthalmol. 2019. https://doi.org/10.1111/ceo.13526.

41. Best UP, Domack H, Schmidt V, Khalifa M. Microinvasive glaucoma surgery-efficacy of trabecular stents in combined interventions: a clinical study on 65 eyes. Ophthalmologe. 2019;116(8):771-9.

42. Clement CI, Howes F, Ioannidis AS, Shiu M, Manning D. One-year outcomes following implantation of second-generation trabecular micro-bypass stents in conjunction with cataract surgery for various types of glaucoma or ocular hypertension: multicenter, multi-surgeon study. Clin Ophthalmol. 2019;13:491-9.

43. The AGIS Investigators. The Advanced Glaucoma Intervention Study (AGIS): 7. The relationship between control of intraocular pressure and visual field deterioration. Am J Ophthalmol. 2000;130(4):429-40.

44. Heijl A, Leske MC, Bengtsson B, Hyman L, Bengtsson B, Hussein M, et al. Reduction of intraocular pressure and glaucoma progression: results from the Early Manifest Glaucoma Trial. Arch Ophthalmol. 2002;120(10):1268-79.

45. Chansangpetch S, Lau K, Perez CI, Nguyen N, Porco TC, Lin SC. Efficacy of cataract surgery with trabecular microbypass stent implantation in combined-mechanism angle closure glaucoma patients. Am J Ophthalmol. 2018;195:191-8.
46. Hernstadt DJ, Cheng J, Htoon HM, Sangtam T, Thomas A, Sng CCA. Case series of combined iStent implantation and phacoemulsification in eyes with primary angle closure disease: 1-year outcomes. Adv Ther. 2019;36(4):976-86.

47. Robin AL, Covert D. Does adjunctive glaucoma therapy affect adherence to the initial primary therapy? Ophthalmology. 2005;112(5):863-8.

48. Nelson P, Aspinall P, Papasouliotis O, Worton B, O'Brien C. Quality of life in glaucoma and its relationship with visual function. J Glaucoma. 2003;12(2):139-50.

49. Chauhan BC, Garway-Heath DF, Goni FJ, Rossetti L, Bengtsson B, Viswanathan AC, et al. Practical recommendations for measuring rates of visual field change in glaucoma. $\mathrm{Br} \mathrm{J}$ Ophthalmol. 2008;92(4):569-73.

50. Bostan C, Harasymowycz P. Episcleral venous outflow: a potential outcome marker for iStent surgery. J Glaucoma. 2017;26(12):1114-9.

51. Fellman RL, Grover DS. Episcleral venous fluid wave: intraoperative evidence for patency of the conventional outflow system. J Glaucoma. 2014;23(6):347-50.

52. Samuelson TW, Chang DF, Marquis R, Flowers B, Lim KS, Ahmed IIK, et al. A Schlemm canal microstent for intraocular pressure reduction in primary open-angle glaucoma and cataract: the HORIZON study. Ophthalmology. 2019;126(1):29-37.

53. Vold S, Ahmed II, Craven ER, Mattox C, Stamper R, Packer $\mathrm{M}$, et al. Two-year COMPASS trial results: supraciliary microstenting with phacoemulsification in patients with open-angle glaucoma and cataracts. Ophthalmology. 2016;123(10):2103-12. 